\title{
Pan-Plasmodium band sensitivity for Plasmodium falciparum detection in combination malaria rapid diagnostic tests and implications for clinical management
}

Michelle L Gatton 1*, Roxanne R Rees-Channer², Jeffrey Glenn ${ }^{3}$, John W Barnwell ${ }^{3}$, Qin Cheng ${ }^{4}$, Peter L Chiodini ${ }^{2,5}$, Sandra Incardona ${ }^{6}$, Iveth J González ${ }^{6}$ and Jane Cunningham7

\begin{abstract}
Background: Malaria rapid diagnostic tests (RDTs) are appropriate for case management, but persistent antigenaemia is a concern for HRP2-detecting RDTs in endemic areas. It has been suggested that pan-pLDH test bands on combination RDTs could be used to distinguish persistent antigenaemia from active Plasmodium falciparum infection, however this assumes all active infections produce positive results on both bands of RDTs, an assertion that has not been demonstrated.

Methods: In this study, data generated during the WHO-FIND product testing programme for malaria RDTs was reviewed to investigate the reactivity of individual test bands against $P$. falciparum in 18 combination RDTs. Each product was tested against multiple wild-type $P$. falciparum only samples. Antigen levels were measured by quantitative ELISA for HRP2, pLDH and aldolase.

Results: When tested against $P$. falciparum samples at 200 parasites/ $\mu \mathrm{L}, 92 \%$ of RDTs were positive; $57 \%$ of these on both the $P$. falciparum and pan bands, while $43 \%$ were positive on the $P$. falciparum band only. There was a relationship between antigen concentration and band positivity; $\geq 4 \mathrm{ng} / \mathrm{mL}$ of HRP2 produced positive results in more than $95 \%$ of P. falciparum bands, while $\geq 45 \mathrm{ng} / \mathrm{mL}$ of pLDH was required for at least $90 \%$ of pan bands to be positive.

Conclusions: In active $P$. falciparum infections it is common for combination RDTs to return a positive HRP2 band combined with a negative pan-pLDH band, and when both bands are positive, often the pan band is faint. Thus active infections could be missed if the presence of a HRP2 band in the absence of a pan band is interpreted as being caused solely by persistent antigenaemia.
\end{abstract}

Keywords: Malaria, Rapid diagnostic test, HRP2, pLDH, Persistent antigenemia

\section{Background}

Antigen-detecting malaria rapid diagnostic tests (RDTs) are an important tool for fever case management and routine malaria surveillance. Available malaria RDTs can detect Plasmodium falciparum only, Plasmodium vivax only, all human Plasmodium species or a combination of species. Combination RDTs typically contain two test bands: a $P$. falciparum-specific test band using either

\footnotetext{
* Correspondence: m.gatton@qut.edu.au

'School of Public Health and Social Work, Queensland University of

Technology, GPO Box 2434, Brisbane Qld 4001, Australia

Full list of author information is available at the end of the article
}

bound anti-histidine rich protein 2 (HRP2) or anti-P. falciparum specific plasmodium lactate dehydrogenase (Pf-pLDH) monoclonal antibodies (Mabs), while the other test band typically detects either all four human Plasmodium species (bound anti-pan specific pLDH or anti-aldolase Mabs) or is species-specific for non-falciparum malaria (against one or more Plasmodium species).

Many studies have reported the performance of HRP2 and pLDH-based RDTs for detecting $P$. falciparum mainly compared to microscopy, as the reference standard [1]. A systematic review of 48 studies describing malaria diagnostic performance that accounted for the imperfect

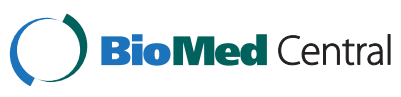

(c) 2015 Gatton et al.; licensee BioMed Central. This is an Open Access article distributed under the terms of the Creative Commons Attribution License (http://creativecommons.org/licenses/by/4.0), which permits unrestricted use, distribution, and reproduction in any medium, provided the original work is properly credited. The Creative Commons Public Domain Dedication waiver (http://creativecommons.org/publicdomain/zero/1.0/) applies to the data made available in this article unless otherwise stated. 
reference standard indicated that although performance varied by species, parasite density and immunity, overall HRP2-detecting RDTs outperformed pLDH-based RDTs and microscopy with high sensitivity and specificity for diagnosing malaria in clinical cases in endemic settings, and also asymptomatic malaria infections in endemic areas [2]. However, HRP2-detecting RDTs are unsuitable for monitoring parasite clearance following anti-malarial treatment due to the persistence of the PfHPR2 antigen in the blood for up to four or five weeks following curative treatment of an infection [1-3].

The issue of persistent antigenaemia in endemic areas has been raised as a factor leading to reduced specificity of HRP2-detecting RDTs for diagnosing acute malaria and over-estimates of malaria prevalence in community surveys [4,5]. Some studies compare HRP2-detecting RDTs to polymerase chain reaction (PCR) and/or microscopy results to distinguish between persistent antigenaemia, and acute infection; and alternatively, it has been suggested that because pLDH is metabolized after three to five days following anti-malarial therapy, that pan-pLDH test bands on combination RDTs could be used to distinguish between persistent antigenaemia (following treatment), and active infection [6]. However, this interpretation is contrary to manufacturers' instructions for HRP2 and pan-pLDH or aldolase-based, combination RDTs, which define a $P$. falciparum infection as either a single HRP2 test line reaction or both HRP2 and pan-test line reactions. Ultimately, any modification to manufacturers' recommendations would only be valid if it can be shown that active infections do not produce the same result (HRP2 test line positive only) as post-treatment antigenaemia.

To investigate the relationships between HRP2 and pan band positivity, the current study analyses the individual band reactivity of $18 \mathrm{P}$. falciparum/pan combination RDT products against samples collected from patients with active $P$. falciparum infection and without history of anti-malarial therapy in the past month. The relationship between test band positivity and antigen concentration in these samples is also described to help explain the observed results.

\section{Methods}

In 2008, an evaluation program to assess the performance of commercially available malaria RDTs was launched by the Western Pacific Regional Office of the World Health Organization (WHO), the Special Programme for Research and Training in Tropical Diseases (TDR) and the Foundation for Innovative New Diagnostics (FIND). Across the first five rounds of product testing conducted between 2008 and 2013, this programme has assessed a total of 210 RDT products, including 63 independent combination products to detect and differentiate $P$. falciparum and non- $P$. falciparum species (hereafter referred to as $\mathrm{Pf}+$ pan products). Data generated during this product testing provides a unique opportunity to assess and compare the performance of RDTs as well as individual test bands on well characterized $P$. falciparum clinical samples.

The full testing protocol is described elsewhere [7]. The specific focus here in is on the results of testing each RDT product against a panel of approximately 100 P. falciparum wild-type parasites at 200 and 2,000 or 5,000 parasites $/ \mu \mathrm{L}$. Over the five rounds of testing a total of 157 parasite samples have been used in these wild-type panels. Each parasite sample was confirmed by PCR to be a mono-infection with $P$. falciparum, and to contain the gene encoding for the PfHRP2 protein. Patients were excluded if they had received any form of anti-malarial therapy in the four weeks preceding enrolment. Antigen levels of each sample were measured by quantitative ELISA for HRP2, pLDH and aldolase [7].

Although a total of $63 \mathrm{Pf}+$ pan products have been tested by the programme, only 18 currently meet the WHO recommended criteria for procurement of malaria RDTs: products that have been tested within the last five years and have high rates of detection $(>75 \%$ panel detection score) of both $P$. falciparum and $P$. vivax at 200 parasites $/ \mu \mathrm{L}$, and low false positive $(<10 \%)$ and invalid $(<5 \%)$ rates [8]. Of these 18 products, the large majority (15) use HRP2 as the target $P$. falciparum band antigen and pan-pLDH as the target antigen for the pan band (Table 1). Raw data for these 18 products was extracted from the full product testing results database for analysis in the current study.

Each product was tested on each $P$. falciparum sample a total of six times; four times using the sample diluted to 200 parasites $/ \mu \mathrm{L}$ and two times using the sample at 2,000 or 5,000 parasites $/ \mu \mathrm{L}$. For each test the band intensity of the control and test bands were graded on a visual scale between 0 and 4 using standard colour charts at the minimum read time recommended by the manufacturer. These results are the basis of this analysis with a band intensity of 0 being classified as negative and intensities of 1,2, 3 or 4 being classified as positive. Any tests which returned a negative result on the control band were classified as invalid and excluded from all analysis. The overall test result was interpreted according to the manufacturer instructions:

1. a positive P. falciparum test band (hereafter referred to as the Pf band) was classified as a positive test for $P$. falciparum, irrespective of the result on the pan test band,

2. a positive result on the pan band when the Pf band was negative was classified as a positive test indicating a non-falciparum infection, and 
Table 1 Combination RDT products included in this study

\begin{tabular}{|c|c|c|c|c|}
\hline Product & Manufacturer & $\begin{array}{l}\text { P. falciparum } \\
\text { band antigen }\end{array}$ & $\begin{array}{l}\text { Pan band } \\
\text { antigen }\end{array}$ & $\begin{array}{l}\text { No. Pf } \\
\text { samples }^{a}\end{array}$ \\
\hline Advantage Malaria Pan + Pf Card - IR231025 & J. Mitra \& Co. Pvt. Ltd. & HRP2 & $\operatorname{Pan}(\mathrm{pLDH})$ & 100 \\
\hline BIOCREDIT Malaria Ag Pf/Pan (HRPII/pLDH) - C30RHA25 & RapiGEN INC. & HRP2 & $\operatorname{Pan}(\mathrm{pLDH})$ & 100 \\
\hline BIONOTE MALARIA P.f.\& Pan Ag Rapid Test Kit - RG19-08 & Bionote,Inc. & HRP2 & $\operatorname{Pan}(\mathrm{pLDH})$ & 99 \\
\hline 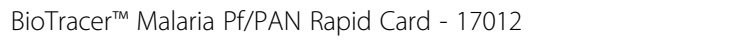 & Bio Focus Co., Ltd. & HRP2 & $\operatorname{Pan}(\mathrm{pLDH})$ & 100 \\
\hline 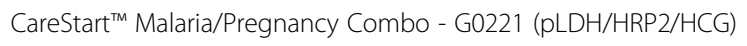 & Access Bio, Inc. & HRP2 & $\operatorname{Pan}(\mathrm{pLDH})$ & 99 \\
\hline 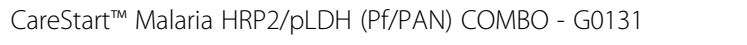 & Access Bio, Inc. & HRP2 & $\operatorname{Pan}(\mathrm{pLDH})$ & 100 \\
\hline CareStart $^{\mathrm{TM} M}$ Malaria pLDH 3 Line Test - G0121 & Access Bio, Inc. & Pf(pLDH) & $\operatorname{Pan}(\mathrm{pLDH})$ & 99 \\
\hline CareStart $^{\mathrm{TM}}$ Malaria Screen - G0231 & Access Bio, Inc. & HRP2 / Pf(pLDH) & $\operatorname{Pan}(\mathrm{pLDH})$ & 99 \\
\hline DIAQUICK Malaria P.f/Pan Cassette - Z11200CE & DIALAB GmbH & HRP2 & $\operatorname{Pan}(\mathrm{pLDH})$ & 100 \\
\hline EzDx ${ }^{\mathrm{TM}}$ Malaria Pan/Pf Rapid Test Detection kit - RK MAL 001 & $\begin{array}{l}\text { Advy Chemical (Affiliate of } \\
\text { Bharat Serums \& Vaccines Ltd.) }\end{array}$ & HRP2 & $\operatorname{Pan}(\mathrm{pLDH})$ & 100 \\
\hline HiSens Malaria Ag Pf/Pv (HRP2/pLDH) Card - HR2923 & HBI Co., Ltd. & HRP2 & $\operatorname{Pan}(\mathrm{pLDH})$ & 100 \\
\hline Humasis Malaria P.f/Pan Antigen Test - AMAL-7025 & Humasis, Co., Ltd. & HRP2 & $\operatorname{Pan}(\mathrm{pLDH})$ & 100 \\
\hline Malaria Pf/Pan One Step Rapid Test - RT 20222 & Zhejiang Orient Gene Biotech Co., Ltd. & HRP2 & $\operatorname{Pan}(\mathrm{pLDH})$ & 100 \\
\hline NanoSign Malaria pf/pan Ag 3.0 - RMAP10 & Bioland Ltd. & HRP2 & $\operatorname{Pan}(\mathrm{pLDH})$ & 98 \\
\hline OnSite Pf/Pan Ag Rapid Test - R0113C & CTK Biotech, Inc. & HRP2 & $\operatorname{Pan}(\mathrm{pLDH})$ & 100 \\
\hline ParaHIT - Total Ver. 1.0 (Device) - 55IC204-10 & Span Diagnostics Ltd. & HRP2 & Aldolase & 98 \\
\hline SD BIOLINE Malaria Ag P.f/Pan - 05FK60/05FK63 & Standard Diagnostics Inc. & HRP2 & $\operatorname{Pan}(\mathrm{pLDH})$ & 100 \\
\hline SD BIOLINE Malaria Ag P.f/Pan - 05FK66 & Standard Diagnostics Inc. & HRP2 & $\operatorname{Pan}(\mathrm{pLDH})$ & 98 \\
\hline
\end{tabular}

${ }^{a}$ Number of $P$. falciparum samples included in the wild type testing panel.

3. a negative result on both test bands was classified as a negative test result indicating no Plasmodium infection.

Since all samples used during testing were known to contain only $P$. falciparum parasites, tests positive for a non-falciparum infection were classified as false positives (non-P. falciparum).

\section{Statistical analysis}

The statistical analysis was conducted using SPSS Statistics Version 21 (IBM). For RDTs testing positive to $P$. falciparum the mean HRP2 band intensities of tests with a positive pan band were compared to those with a negative pan band using a paired t-test, with the RDT product used as the basis for pairing. Pearson's correlation was used as the measure of association between HRP2 and pLDH antigen levels within parasite samples.

\section{Results}

\section{Test and band positivity}

A total of 7,160 and 3,580 tests were conducted using the 18 RDT products and $P$. falciparum samples containing 200 parasites $/ \mu \mathrm{L}$ and 2,000 or 5,000 parasites $/ \mu \mathrm{L}$, respectively. These tests yielded 7,146 and 3,574 valid RDT results for the low and high parasite concentrations, respectively.
When tested against 200 parasites $/ \mu \mathrm{L}, 92.3 \%$ of the tests returned a positive result for $P$. falciparum, $0.9 \%$ returned a false positive result for a non-falciparum infection and $6.7 \%$ of tests were negative. Of the tests that were positive for $P$. falciparum infection using the low parasite density samples, $57.1 \%$ were positive on both the Pf and pan bands, while $42.9 \%$ were positive on the Pf band only. The proportion of tests positive for $P$. falciparum varied between individual RDT products (range $81.8 \%$ to $96.9 \%$, Figure 1 ). There was also considerable product variability in the proportion of positive RDTs with a positive pan band (range $25.0 \%$ to $95.9 \%$, Figure 1).

When tested using parasites at the higher concentration of 2,000 or 5,000 parasites $/ \mu \mathrm{L}, 99.7 \%$ of tests were positive for $P$. falciparum with $96.7 \%$ of these tests returning a positive Pf and pan band. False positive results for a nonfalciparum infection occurred in $0.2 \%$ of tests, while $0.1 \%$ of tests were negative for Plasmodium.

\section{Band intensities}

The mean band intensity for tests positive on the Pf band at 200 parasites $/ \mu \mathrm{L}$ was 2.47 (standard deviation (sd) 0.96) for the HRP2-detecting Pf bands and 2.84 (sd 1.03) for PfpLDH-detecting Pf bands. In contrast, the mean intensity for positive pan bands was lower at 1.50 (sd 0.78 ), with $65 \%$ of tests having a pan band intensity of 1 . All band intensities increased when products 


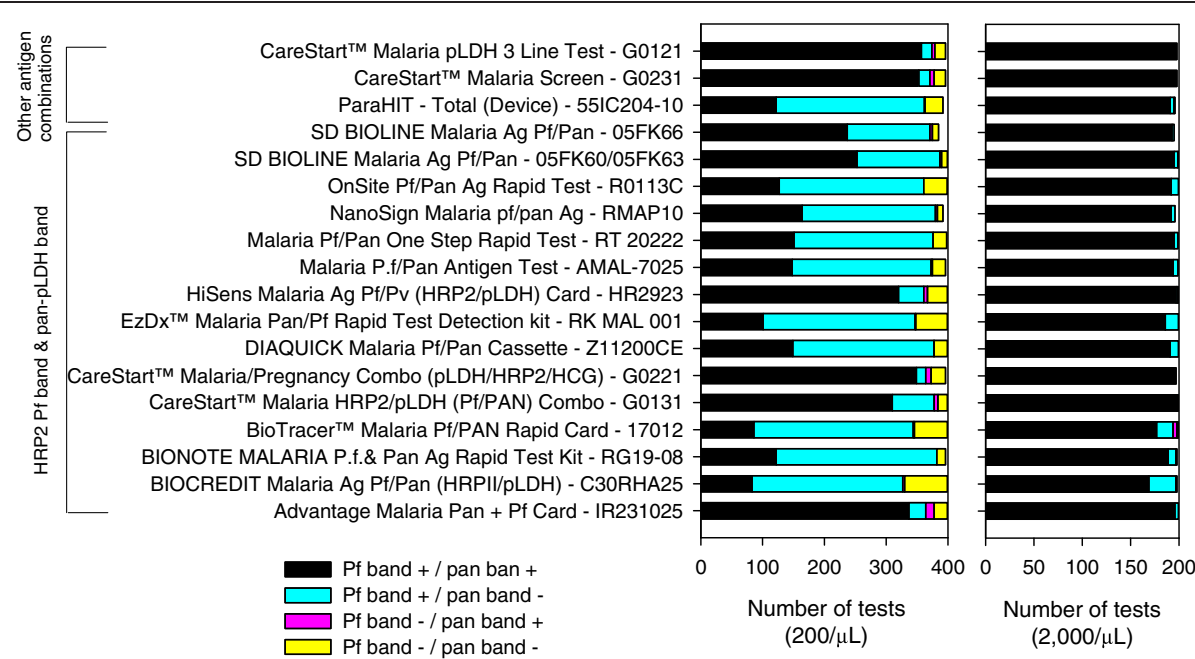

Figure 1 Performance of individual test bands of RDT products when tested using wild-type $P$. falciparum parasites at densities of 200 parasites/ $\mu \mathrm{L}$ and 2,000 parasites $/ \mu \mathrm{L}$.

were tested using samples containing 2,000 or 5,000 parasites $/ \mu \mathrm{L}$; mean band intensities were 3.68 (sd 0.63), 3.91 (sd 0.32) and 2.43 (sd 0.98) for the HRP2-detecting Pf bands, PfpLDH-detecting Pf bands and the pan bands, respectively. At this higher parasite density $16.1 \%, 42.0 \%$, $23.5 \%$ and $18.4 \%$ of RDTs returning positive results on both test bands had pan band intensities of 1, 2, 3 and 4, respectively.

A direct comparison of the pan and Pf band intensities of individual tests at the lower parasite density revealed that in $0.9 \%, 12.7 \%$ and $86.4 \%$ of tests the pan band had a greater, the same, or less intensity than the Pf band, respectively. For the 5,720 tests which had a higher Pf band intensity compared to the pan band intensity, the difference in band intensities was one unit for $39.2 \%$ of tests, two units in $37.8 \%$ of tests and three units in $19.1 \%$ of tests.

The mean band intensities for positive RDTs with HRP2-detecting bands varied by product with values ranging from 1.98 (BioTracer ${ }^{\mathrm{ms}}$ Malaria Pf/PAN Rapid Card - 17012) to 3.21 (BIONOTE MALARIA P.f.\& Pan Ag Rapid Test Kit - RG19-08) when tested against 200 parasites $/ \mu \mathrm{L}$ (Figure 2). Product means were approximately normally distributed around the overall mean of 2.47 . The mean intensities for positive pan bands also varied by product ranging from 1.01 (EzDx RK MAL 001) to 2.26 (Carestart G0231), but the distribution was positively skewed with $50 \%$ of the products having a mean band intensity less than 1.19 and only three products (Carestart G0221, Carestart G0121 and Carestart G0231) having means greater than 2.1.

A more focused analysis of band intensity was conducted for RDTs with a HRP2-detecting Pf band using the results of testing with samples containing 200 parasites $/ \mu \mathrm{L}$. For each product the mean and distribution of the Pf band intensity was calculated for tests that were positive on both the Pf and pan bands and those that were positive only on the Pf band (Figure 3). Comparison of the mean values revealed that on average, the Pf band intensity for a product was 0.73 units (95\% CI: 0.53-0.93 units) higher when both the Pf and pan bands were positive $($ mean $=2.71$, sd 0.39 ) compared to those where only the Pf band was positive $\left(\right.$ mean $=1.97$, sd 0.48) (paired t-test, $\left.\mathrm{t}_{16}=-7.63, \mathrm{p}<0.001\right)$.

Relationship of antigen concentrations in wild-type Plasmodium falciparum samples and positivity of test bands The $P$. falciparum samples used in the testing of the 18 RDTs had varying antigen concentrations. When the

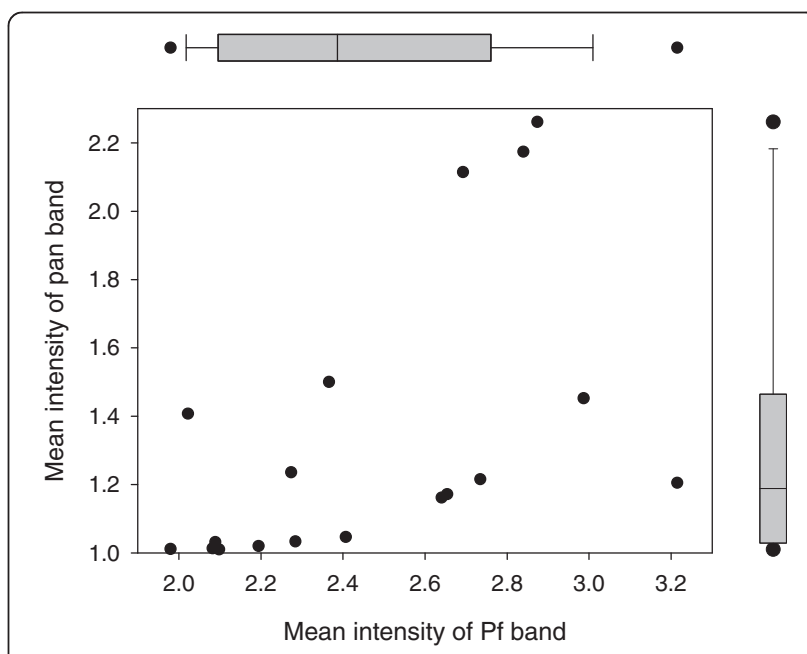

Figure 2 Mean band intensities for 18 RDT products when tested against wild-type $P$. falciparum parasites at 200 parasites $/ \mu \mathrm{L}$. The overall distribution of the Pf and pan band intensity is illustrated by the corresponding box plot; box extends from first to third quartile, with line indicating median. 


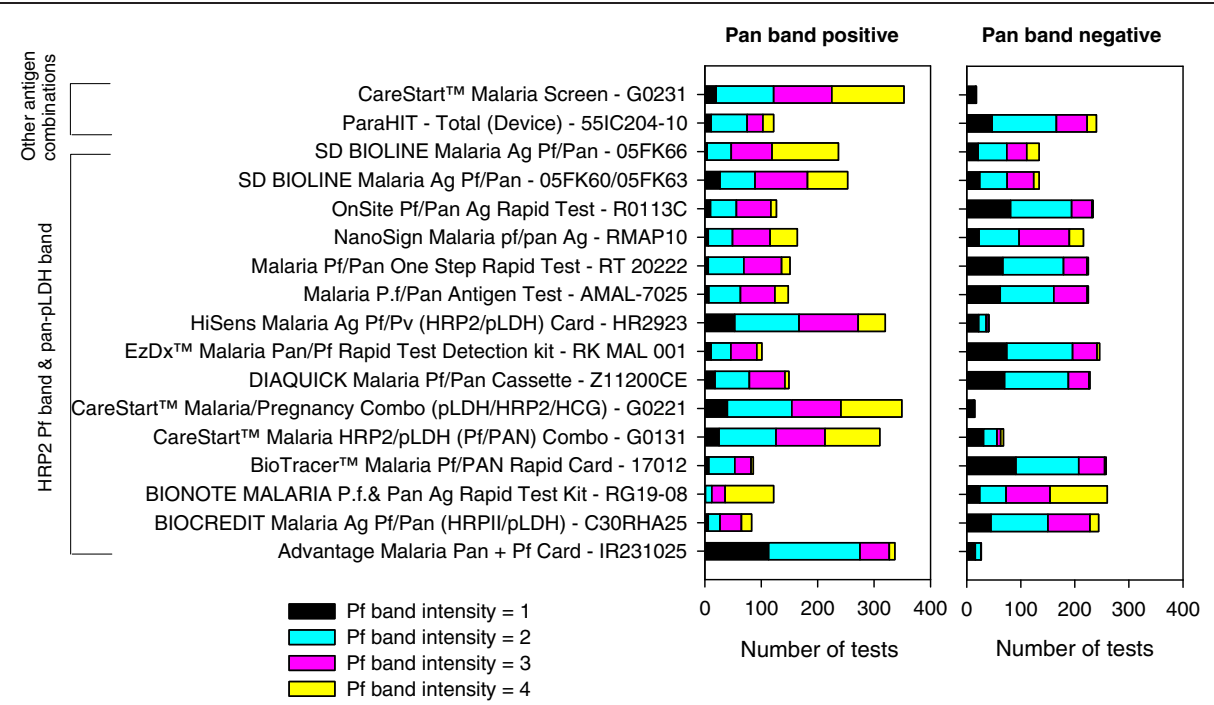

Figure 3 Distribution of Pf band intensities for HRP2-detecting combination RDTs returning a positive result against samples containing $200 \mathrm{P}$. falciparum parasites/ $\boldsymbol{\mu L}$. Tests are grouped based on whether the pan band was positive (left) or negative (right) in the individual tests.

data for both the low parasite density $(200 \mathrm{p} / \mathrm{ul})$ and the high density $(2,000$ or $5,000 \mathrm{p} / \mathrm{ul})$ samples were pooled, the HRP2 concentrations ranged from $0.62 \mathrm{ng} / \mathrm{mL}$ to $820.2 \mathrm{ng} / \mathrm{mL}$, while $\mathrm{pLDH}$ concentrations ranged from $0.19 \mathrm{ng} / \mathrm{mL}$ to $1,800 \mathrm{ng} / \mathrm{mL}$ (Figure 4). The HRP2 and pLDH concentrations within individual samples were significantly correlated $(\mathrm{r}=0.45, \mathrm{p}<0.001)$.

There was a clear relationship between antigen concentration and band positivity rate (Figure 5). With the exception of two samples, HRP2 concentrations of $4 \mathrm{ng} / \mathrm{mL}$ or greater produced positive results in over $95 \%$ of HRP2-detecting test bands. Below this threshold the

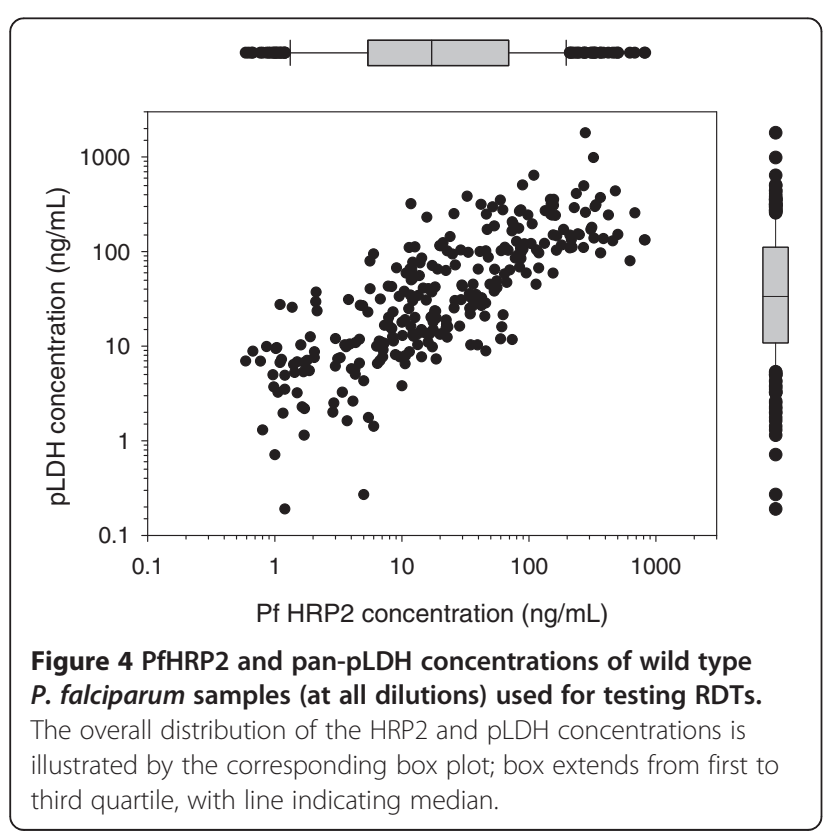

test band positivity was highly variable showing a rapid decline with concentration.

A similar pattern was observed for pLDH and pLDH-detecting test bands; pLDH concentrations above $45 \mathrm{ng} / \mathrm{mL}$ returned positive pLDH test bands in over $90 \%$ of tests. Below this threshold the positivity of the pLDH test bands declined with concentration (Figure 5).

\section{Discussion}

The HRP2 antigen is known to persist within the circulation following curative treatment and this has led to the suggestion that HRP2-detecting RDTs have reduced specificity to detect active malaria infection in moderate to high transmission areas. On the other hand, pLDH is metabolized more quickly and, therefore, RDTs detecting this enzyme are expected to revert to negative more quickly following malaria treatment. Therefore, a tempting strategy to differentiate past, treated infections from current infections is to use combined HRP2 and pan detecting RDTs, or RDTs that contain separate HRP2 and Pf-pLDH test bands. While this approach initially sounds reasonable in circumstances where a recent infection has occurred [9], its general application to clinical management of fever cases is subject to discussion. Hawkes et al. [6] conclude that requiring positivity of both the HRP2 and pLDH bands in a combination RDT can improve diagnostic specificity for falciparum malaria in a sub-Saharan African context, by excluding false positive HRP2 results due to persistent antigenaemia. This conclusion was reached after comparing RDT results to microscopy in a sample of children under five years of age hospitalized for febrile illness. While this suggestion may be appropriate for selection of individuals for clinical trials, the extension of 


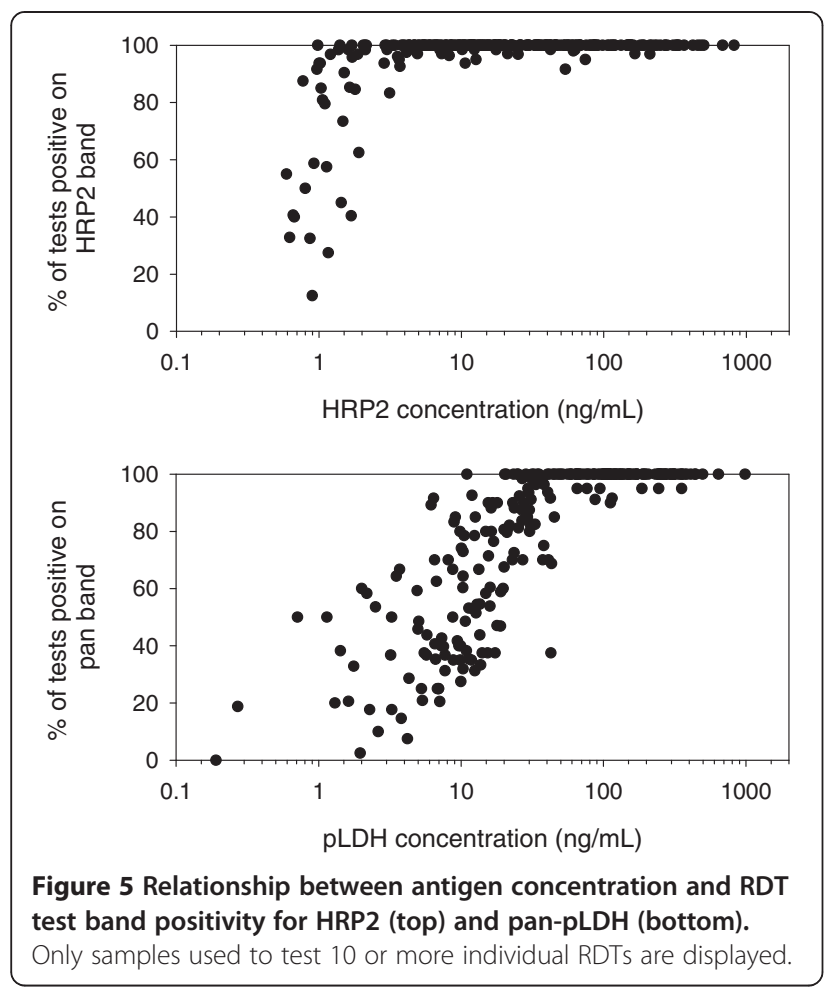

this to clinical diagnosis or management is questionable since this conclusion is based on the assumption that all RDTs that are HRP2-positive but pLDH-negative represent persistent antigenaemia. Underpinning this assumption is the premise that all current infections produce positive results on both the HRP2 and pLDH bands of the RDTs, an assertion that has not been tested previously in a systematic way for multiple RDT products.

To address this gap this study analysed data generated during the WHO-FIND product testing programme for malaria RDTs with a specific focus on the reactivity of individual test bands in 18 combination malaria RDTs that meet the current WHO recommended procurement criteria. Seventeen of these products used PfHPR2 for the detection of P. falciparum. During WHO-FIND product testing, these 18 products were able to consistently detect $>75 \%$ of the wildtype $P$. falciparum and $P$. vivax samples at a dilution of 200 parasites $/ \mu \mathrm{L}$ with low false positive rates or incorrect species identification. Although this study focused on the detection of P. falciparum, the RDTs selected for inclusion in this analysis were purposely restricted to those meeting the criteria for both $P$. falciparum and $P$. vivax detection to be assured that the pan test bands were performing well when interpreted according to the manufacturer's instructions. This removes the possibility that the results observed in this study were caused by a dysfunctional pan band.
The results of the current analysis indicate that there is a difference in the sensitivity of the HRP2-detecting test bands and pan-pLDH test bands in the detection of active infection. At low parasite densities it was observed that the HRP2-detecting band returned a positive result in the absence of a positive pan band in over $40 \%$ of positive tests, with this percentage being product specific. This trend was less evident at higher parasite densities where both bands tended to be positive simultaneously. This result matches that previously reported for the SD BIOLINE Malaria Ag P.f/Pan (catalogue number 05FK60, Standard Diagnostics Inc., South Korea) product where the proportion of tests positive on both the HPRP2 and pLDH bands progressively increased with parasite density from $6.7 \%$ at $<100$ parasites $/ \mu \mathrm{L}$ to $98.5 \%$ at $>1000$ parasites $/ \mu \mathrm{L}$ [10]. Similar patterns were also reported for the SD Malaria Antigen P.f (catalogue number 05FK90, Standard Diagnostics Inc. South Korea) product [11].

The analysis also revealed that even when both the pan and P. falciparum bands were positive, the band intensity of the pan band was generally lower than the Pf band, irrespective of the parasite density. At the lower parasite density of 200 parasites/ $\mu \mathrm{L}, 65 \%$ of the positive pan bands had an intensity of 1 . This proportion reduced to $16 \%$ when samples contained 2,000 or 5,000 parasites/ $\mu \mathrm{L}$. Thus even when the pan band is positive, it is often faint. The RDT testing conducted for the WHO-FIND product testing programme occurs at the $\mathrm{CDC}$ in ideal conditions which assists with the identification of these weak positive results. However faint bands are often difficult to see and may well be missed by health workers working in reduced lighting conditions or with reduced visual acuity $[12,13]$. This would inflate the proportion of RDTs having a positive HRP2 band and negative pan band.

There are several possible reasons for the observed differences in positivity and intensity of HRP2 Pf bands compared to $\mathrm{pLDH}$ pan bands. First, the relative abundance of HRP2 and pLDH may differ within parasites. The samples used within the current study had wide but similar ranges of concentrations for HRP2 and pLDH and there was a significant positive correlation between the two antigen concentrations. Hence it does not appear that gross differences in antigen concentration were the cause of the disparity in test band performance against $P$. falciparum.

The second possible reason is related to differences in avidity of the antigen for binding the antibodies bound to the RDT test lines. The current results indicate that approximately $4 \mathrm{ng} / \mathrm{mL}$ of HRP2 is required to obtain a positive HRP2 band in over $95 \%$ of tests, compared to over $45 \mathrm{ng} / \mathrm{mL}$ for pan-pLDH. This difference in concentration aligns with the HRP2 antigen having multiple binding epitopes due to its repeat structure, compared to pLDH, which is a single epitope. Lee et al. [14] reported the most 
frequent HPR2 motifs occurred within the HRP2 sequence with a frequency of $8-25$, depending on the motif and specific sequence. This frequency aligns approximately with the differences in threshold concentration observed here. Thus, it appears that the difference in antibodybinding avidity between HRP2 and PLDH may be a cause of differences in sensitivity of the respective test bands. It is unlikely that the ordering of the bands on the strip accounts for diminished performance of the pan test bands because for all except two tests included in this analysis the pan test band is the farthest from the origin and this position is advantageous as the flow rate at which the analyte passes the capture reagent line is slower; and the effective concentration of analyte in the sample is higher [15].

Many studies have been conducted to assess the performance of specific malaria RDTs in different settings [1]. However few have directly compared HRP2-detecting RDTs to pLDH-detecting RDTs, and examined potential reasons for the differences in the two types of tests. A longitudinal study in Uganda identified that HRP2detecting RDTs provide better detection of parasites at low densities compared to pLDH-detecting RDTs, but have lower specificity due to the slower clearance of HRP2 antigenaemia from the blood circulation [16]. Differences have also been observed in performance between regions with different transmission intensities, and this difference was attributed to the superior ability of HRP2-detecting RDTs over pLDH-detecting RDTs to detect sub-patent parasitaemia [17]. These results, although obtained by comparing different RDTs that both detect $P$. falciparum, appear relevant for the combination tests with antibodies against these two antigens. It would be possible to distinguish between persistent HRP2 antigenaemia and viable falciparum parasitaemia in a given blood sample by comparing the results of an HRP2-based RDT with a separate, equally well-performing RDT containing a falciparum pLDH band, but that is not a viable proposition for field use.

Therefore, the problem remains of how to clinically manage a febrile patient with a history of recent antimalarial treatment who returns a positive HRP2 band, but a negative pLDH band on a combination RDT. This can result from persistent antigenaemia or malaria re-infection or recrudescence (treatment failure). Treatment failure may result from drug resistance or inadequate exposure to the drug due to sub-optimal dosing, poor adherence, vomiting, unusual pharmacokinetics in an individual or substandard medicines. It is important to determine from the patient's history whether he or she vomited the previous treatment or did not complete the full course of treatment. These cases need to be treated again with the artemisinin-combination therapy (ACT) recommended as first-line in the area.
If the patient's history reveals that he/she has taken the full and correctly dosed treatment course, the possibility of true treatment failure can only be excluded by referring the patient to a facility with good quality microscopy. Referral may be necessary anyway to obtain second-line treatment. In individual patients, it may not be possible to distinguish recrudescence from re-infection, although lack of resolution of fever and parasitaemia (on microscopy) or their recurrence within four weeks of treatment are considered failures of treatment with the currently recommended ACT. For these cases the recommended second-line treatment is an alternative ACT known to be effective in the region. In addition to the above guidance, in all cases the health provider should always consider other diagnoses and follow closely for a clinical response.

Recurrence of fever and parasitaemia more than four weeks after treatment may be due to either recrudescence or a new infection. The distinction can only be made by genotyping of parasites from the initial and the recurrent infections. As parasite genotyping is not routinely used in patient management, then all presumed treatment failures after four weeks of initial treatment should be considered new infections and be treated with the first-line ACT.

Ultimately, the results from this study clearly show that in the setting of active (untreated) malaria infection, it is common for HRP2/pan-pLDH combination tests to return a positive HRP2 band combined with a negative pan-pLDH band at low parasite densities, and when both bands are positive, often the pan band is faint even at densities of 2,000 parasites/ $\mu \mathrm{L}$. Therefore it would be dangerous to interpret the presence of a HRP2 band in the absence of a pan band as being caused solely by persistent antigenaemia in a clinical setting. Only when the sensitivity of the pLDH-detecting pan band is improved to have the comparable reactivity as the HRP2 band for detecting P. falciparum could persistent antigenaemia be confidently attributed as the cause of HRP2-positive, pan-negative RDT results.

\footnotetext{
Competing interests

The authors do not have a commercial or other association that might pose a conflict of interest. The opinions expressed herein are those of the authors and do not necessarily reflect those of the Australian Defence Force Joint Health Command or the World Health Organization or the Centers for Disease Control and Prevention.
}

\section{Authors' contributions}

MLG conceived the study, performed the data analysis and drafted the manuscript. RRR carried out the ELISA. JG conducted the RDT testing and performed data entry. JWB participated in the study design and coordination. QC participated in the study design and coordination and helped to draft the manuscript. PLC participated in the study design and coordination. SI participated in the study design and collation of the data. IJG participated in the study design and coordination. JC participated in the study design and coordination, and assisted with drafting the manuscript. All authors read and approved the final manuscript. 


\section{Acknowledgements}

The authors would like to thank Dr Andrea Bosman for critical review of the manuscript.

\section{Author details}

${ }^{1}$ School of Public Health and Social Work, Queensland University of Technology, GPO Box 2434, Brisbane Qld 4001, Australia. ²National Institute for Health Research University College London Hospitals Biomedical Research Centre, Hospital for Tropical Diseases, London, UK. ${ }^{3}$ Malaria Branch, Division of Parasitic Diseases and Malaria, Centers for Disease Control and Prevention, Center for Global Health, Atlanta, USA. ${ }^{4}$ Drug Resistance and Diagnostics, Australian Army Malaria Institute, Brisbane, Australia. ${ }^{5}$ London School of Hygiene and Tropical Medicine, London, UK. ${ }^{6}$ FIND (Foundation for Innovative New Diagnostics), Geneva, Switzerland. ${ }^{7}$ Global Malaria Programme, World Health Organization, Geneva, Switzerland.

Received: 15 December 2014 Accepted: 1 March 2015 Published online: 18 March 2015

\section{References}

1. Abba K, Deeks JJ, Olliaro P, Naing CM, Jackson SM, Takwoingi Y, et al. Rapid diagnostic tests for diagnosing uncomplicated $P$. falciparum malaria in endemic countries. Cochrane Database Syst Rev. 2011;7:CD008122.

2. Ochola LB, Vounatsou P, Smith T, Mabaso ML, Newton CR. The reliability of diagnostic techniques in the diagnosis and management of malaria in the absence of a gold standard. Lancet Infect Dis. 2006;6:582-8.

3. Wilson ML. Malaria rapid diagnostic tests. Clin Infect Dis. 2012;54:1637-41.

4. Samadoulougou S, Kirakoya-Samadoulougou F, Sarrassat S, Tinto H, Bakiono F, Nebie I, et al. Paracheck(R) rapid diagnostic test for detecting malaria infection in under five children: a population-based survey in Burkina Faso. Malar J. 2014;13:101

5. Keating J, Miller JM, Bennett A, Moonga HB, Eisele TP. Plasmodium falciparum parasite infection prevalence from a household survey in Zambia using microscopy and a rapid diagnostic test: implications for monitoring and evaluation. Acta Trop. 2009:112:277-82.

6. Hawkes M, Conroy AL, Opoka RO, Namasopo S, Liles WC, John CC, et al. Use of a three-band HRP2/pLDH combination rapid diagnostic test increases diagnostic specificity for falciparum malaria in Ugandan children. Malar J. 2014;13:43.

7. $\mathrm{WHO}, \mathrm{FIND}, \mathrm{CDC}$. Malaria rapid diagnostic test performance: results of WHO product testing of malaria RDTs: round 5 (2013). Geneva, Switzerland: World Health Organization; 2014.

8. WHO Global Malaria Programme: Information note on recommended selection criteria for procurement of malaria rapid diagnostic tests. 2014; WHO Report: WHO/HTM/GMP/2014.8.

9. Nyunt MH, Kyaw MP, Win KK, Myint KM, Nyunt KM. Field evaluation of HRP2 and pan pLDH-based immunochromatographic assay in therapeutic monitoring of uncomplicated falciparum malaria in Myanmar. Malar J. 2013;12:123.

10. Van der Palen M, Gillet P, Bottieau E, Cnops L, Van Esbroeck M, Jacobs J. Test characteristics of two rapid antigen detection tests (SD FK50 and SD FK60) for the diagnosis of malaria in returned travellers. Malar J. 2009;8:90.

11. Heutmekers M, Gillet P, Cnops L, Bottieau E, Van Esbroeck M, Maltha J, et al. Evaluation of the malaria rapid diagnostic test SDFK90: detection of both PfHRP2 and Pf-pLDH. Malar J. 2012:11:359.

12. Counihan H, Harvey SA, Sekeseke-Chinyama M, Hamainza B, Banda R, Malambo T, et al. Community health workers use malaria rapid diagnostic tests (RDTs) safely and accurately: results of a longitudinal study in Zambia. Am J Trop Med Hyg. 2012;87:57-63.

13. Mukadi P, Gillet P, Lukuka A, Mbatshi J, Otshudiema J, Muyembe JJ, et al. External quality assessment of reading and interpretation of malaria rapid diagnostic tests among 1849 end-users in the Democratic Republic of the Congo through Short Message Service (SMS). PLoS One. 2013;8:e71442.

14. Lee N, Gatton ML, Pelecanos A, Bubb M, Gonzalez I, Bell D, et al. Identification of optimal epitopes for Plasmodium falciparum rapid diagnostic tests that target histidine-rich proteins 2 and 3. J Clin Microbiol. 2012;50:1397-405.

15. Millipore Corporation: Rapid lateral flow test strips: consideration for product development. Lit. No. TB500EN00 2002, Bedford MA.
16. Hopkins H, Kambale W, Kamya MR, Staedke SG, Dorsey G, Rosenthal PJ. Comparison of HRP2- and pLDH-based rapid diagnostic tests for malaria with longitudinal follow-up in Kampala, Uganda. Am J Trop Med Hyg. 2007;76:1092-7.

17. Hopkins H, Bebell L, Kambale W, Dokomajilar C, Rosenthal PJ, Dorsey G. Rapid diagnostic tests for malaria at sites of varying transmission intensity in Uganda. J Infect Dis. 2008;197:510-8.

\section{Submit your next manuscript to BioMed Central and take full advantage of:}

- Convenient online submission

- Thorough peer review

- No space constraints or color figure charges

- Immediate publication on acceptance

- Inclusion in PubMed, CAS, Scopus and Google Scholar

- Research which is freely available for redistribution 